Mathematical Modelling AND ANALysis

Volume 17 Number 2, April 2012, 217-226

http://dx.doi.org/10.3846/13926292.2012.661697

(c) Vilnius Gediminas Technical University, 2012
Publisher: Taylor\&Francis and VGTU

http://www.tandfonline.com/TMMA

Print ISSN: 1392-6292

Online ISSN: 1648-3510

\title{
Nonlinear Problems with Asymmetric Principal Part
}

\section{Armands Gritsans and Felix Sadyrbaev}

\author{
Daugavpils University \\ Parades str. 1, LV-5400, Daugavpils, Latvia \\ E-mail(corresp.): armands.gricans@du.lv \\ E-mail: felix@latnet.lv
}

Received September 6, 2011; revised January 13, 2012; published online April 1, 2012

Abstract. The boundary value problem

$$
x^{\prime \prime}=-\lambda f\left(x^{+}\right)+\mu f\left(x^{-}\right)+h\left(t, x, x^{\prime}\right), \quad x(0)=0=x(1)
$$

is considered provided that $f:[0,+\infty) \rightarrow[0,+\infty)$ is Lipschitzian and $h:[0,1] \times \mathbb{R} \times$ $\mathbb{R} \rightarrow \mathbb{R}$ is continuous and Lipschitzian in $x$ and $x^{\prime}$. We assume that $f$ is bounded by two linear functions $k x$ and $l x$, where $k>l>0$, and $h$ is bounded. We find the conditions on $(\lambda, \mu)$ which guarantee the existence of a solution to the problem. These conditions are of geometrical nature.

Keywords: nonlinear spectra, Fučík spectrum, comparison, angular functions, Dirichlet boundary value problem.

AMS Subject Classification: 34B08; 34B15.

\section{Introduction}

There is intensive literature on boundary value problems for the second order ordinary differential equations which depend on two parameters, for example $[1,2,3,5,7,10,11,12]$. A special class of problems deals with the so called asymmetric equations. The classical representative of such equations is the Fučík equation

$$
x^{\prime \prime}=-\lambda x^{+}+\mu x^{-}, \quad x^{+}=\max \{x, 0\}, x^{-}=\max \{-x, 0\}, \lambda>0, \mu>0,
$$

which is usually considered together with some boundary conditions, for instance, the Dirichlet ones $x(0)=0, x(1)=0$.

The results on Fučík problem can be used for investigation of essentially nonlinear problems of the type

$$
x^{\prime \prime}+g(x)=h\left(t, x, x^{\prime}\right), \quad x(0)=0, \quad x(1)=0,
$$


where the ratio $g(x) / x$ tends to finite limits as $x \rightarrow \pm \infty$ and $h$ is bounded. These limits (as points in $\mathbb{R}^{2}$ ) have to be separated from the Fučík spectrum, so that the problem had a solution.

There were attempts $[4,5,6]$ to consider Fučík type equations of the form

$$
x^{\prime \prime}=-\lambda f\left(x^{+}\right)+\mu g\left(x^{-}\right),
$$

where $f$ and $g$ are (nonlinear) positively valued functions.

In this paper we consider the boundary value problem

$$
x^{\prime \prime}=-\lambda f\left(x^{+}\right)+\mu f\left(x^{-}\right)+h\left(t, x, x^{\prime}\right), \quad x(0)=0, \quad x(1)=0,
$$

where $f$ is a positive valued function such that $f(0)=0$ and $h$ is a bounded nonlinearity. Functions $f$ and $h$ are such that there is a unique solvability of the Cauchy problems and continuous dependence of solutions on the initial data.

We suppose that

$$
l x<f(x)<k x, \quad \forall x>0,0<l<k .
$$

If $f(x)$ is a linear function (i.e., $f(x)=k x$ ) then the two-parameter problem is given as

$$
x^{\prime \prime}=-\lambda k x^{+}+\mu k x^{-}+h\left(t, x, x^{\prime}\right), \quad x(0)=0, \quad x(1)=0 .
$$

It is known [3] that this problem is solvable for any bounded nonlinearity $h$ if $(\lambda k, \mu k)$ belongs to "good" regions in the first quadrant of $(\lambda, \mu)$-plane. We discuss this below.

If the principal part looks like in problem

$$
x^{\prime \prime}=-\lambda f\left(x^{+}\right)+\mu g\left(x^{-}\right)+h\left(t, x, x^{\prime}\right), \quad x(0)=0, \quad x(1)=0,
$$

then analysis of it becomes more complicated. There are some results which state that the reduced problem

$$
x^{\prime \prime}=-\lambda f\left(x^{+}\right)+\mu g\left(x^{-}\right), \quad x(0)=0, \quad x(1)=0,
$$

is non-trivially solvable if $(\lambda, \mu)$ belongs to solution surfaces [8, 11]. If $g(x)$ is bounded between two linear functions (like function $f(x)$ ), then similar results can be obtained. The aim of this paper is to present the existence results for the problem (1.1).

\section{Quasi-Linear Fučík Problem}

Consider the problem

$$
x^{\prime \prime}=-\lambda x^{+}+\mu x^{-}+h\left(t, x, x^{\prime}\right), \quad x(0)=0, \quad x(1)=0 .
$$

In order to formulate the existence conditions we need first to consider the Fučík spectrum. The Fučík spectrum $\Sigma_{F}$ is a set of points $(\lambda, \mu)$ such that the problem

$$
x^{\prime \prime}=-\lambda x^{+}+\mu x^{-}, \quad x(0)=0, \quad x(1)=0
$$




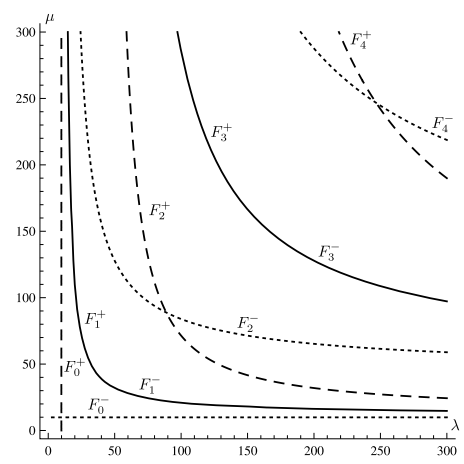

Figure 1. The Fučík spectrum.

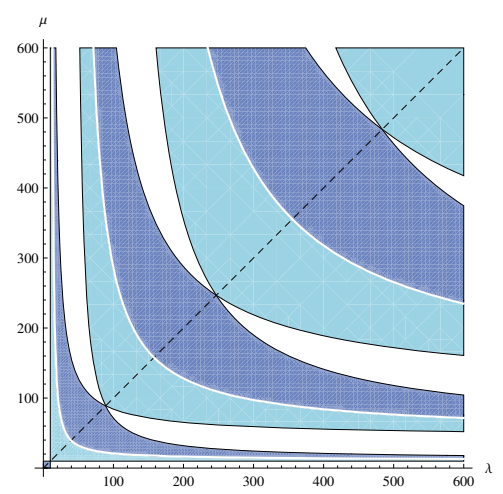

Figure 2. "Good" regions shaded; light shaded for solutions with $2 n-1$ zeros in $(0,1)$, dark shaded for solutions with $2 n$ zeros in $(0,1), n=1,2, \ldots$; "bad" regions white.

has a nontrivial solution $x(t ; \lambda, \mu)$. The Fučík spectrum $\Sigma_{F}$ consists of a set of curves located in the first quadrant of $(\lambda, \mu)$-plane [3] (see, Fig. 1).

If $(\lambda, \mu) \notin \Sigma_{F}$ then the problem (2.2) has only the trivial solution. This is insufficient for solvability of the problem (2.1) for any bounded $h$. The solvability, however, can be guaranteed for "good" regions.

Since it is essential for proving of the main result, we discuss solvability of the problem (2.1). Consider the Cauchy problem

$$
x^{\prime \prime}=-\lambda x^{+}+\mu x^{-}+h\left(t, x, x^{\prime}\right), \quad x(0)=0, \quad x^{\prime}(0)=\alpha,
$$

where $h$ is bounded. Introduce the functions $u(t)$ and $v(t)$ as solutions of the Cauchy problems

$$
\begin{aligned}
& u^{\prime \prime}=-\lambda u^{+}+\mu u^{-}, \quad u(0)=0, \quad u^{\prime}(0)=1, \\
& v^{\prime \prime}=-\lambda v^{+}+\mu v^{-}, \quad v(0)=0, \quad v^{\prime}(0)=-1 \text {. }
\end{aligned}
$$

Let $x(t ; \alpha)$ be a solution of (2.3). The normalized functions $y(t ; \alpha)=x(t ; \alpha) / \alpha$ tend respectively to the functions $u(t)$ and $v(t)$ as $\alpha \rightarrow \pm \infty$. Notice that $y(t ; \alpha)$ satisfies also the equation

$$
y^{\prime \prime}=-\lambda y^{+}+\mu y^{-}+h\left(t, x, x^{\prime}\right) / \alpha,
$$

where $h\left(t, x, x^{\prime}\right) / \alpha$ tends to zero uniformly in $t, x, x^{\prime}$ as $\alpha \rightarrow \infty$. If the condition $y(1 ;+\infty) y(1 ;-\infty)<0$ is satisfied, which is equivalent to

$$
u(1) v(1)<0,
$$

then the existence of $x\left(t ; \alpha_{0}\right)$ which solves the problem $(2.1)$ can be concluded. Therefore problem $(2.1)$ is solvable if $(\lambda, \mu)$ is not in $\Sigma_{F}$ but condition (2.4) holds. The regions of $(\lambda, \mu)$-plane where $u(1) v(1)<0$ are shaded in Fig. 2 . 
So if $(\lambda, \mu)$ are in the shaded region but not in the Fuč́k spectrum then the problem $(2.1)$ is solvable for any bounded $h\left(t, x, x^{\prime}\right)$. If $h(t, 0,0) \not \equiv 0$ then there exists a non-trivial solution.

If precise location of a point $(\lambda, \mu)$ between definite branches of the Fučík spectrum is given, then we can state the existence of a solution with definite nodal structure.

\section{The Problem}

Consider the problem (1.1), where $f(x)$ is such that (1.2) fulfils. To formulate the existence result we need to consider two auxiliary problems

$$
\begin{aligned}
& x^{\prime \prime}=-\lambda k x^{+}+\mu k x^{-}, \quad x(0)=0, \quad x(1)=0, \\
& x^{\prime \prime}=-\lambda l x^{+}+\mu l x^{-}, \quad x(0)=0, \quad x(1)=0 .
\end{aligned}
$$

Denote the spectra of these problems $\Sigma_{F}(k)$ and $\Sigma_{F}(l)$ respectively. Both spectra have "good" regions. Let $D(k)_{i}$ be a part of "good" region where solutions of the IVPs $(3.1), x(0)=0, x^{\prime}(0)= \pm 1$ have exactly $i$ zeros in $(0,1)$. In "good" regions these two solutions also are of opposite signs at $t=1$ and this is important.

Similarly regions $D(l)_{i}$ are introduced.

Notice that the spectrum $\Sigma_{F}(k)$ (and $\Sigma_{F}(l)$ ) can be obtained from the Fučík spectrum $\Sigma_{F}$ by compression (if $k>1$ ) or by extension (if $0<k<1$ ).

Theorem 1. Suppose that $f:[0,+\infty) \rightarrow[0,+\infty)$ is Lipschitzian and $l x<$ $f(x)<k x$ for $x>0,0<l<k$. Assume that $h:[0,1] \times \mathbb{R}^{2} \rightarrow \mathbb{R}$ is continuous and satisfy the Lipschitz condition in $x$ and $x^{\prime}$. Let $(\lambda, \mu)$ be in $D_{i}=D(k)_{i} \cap D(l)_{i}$ for some $i \in\{0,1, \ldots\}$. Then provided that $h$ is bounded the problem (1.1) has a solution.

To prove the theorem, we need some comparison results which we consider in separate subsections.

\subsection{Differential inequality}

The following assertion is a slight modification of Theorem 14.1 in [9].

Theorem 2. Let $\varphi(t)$ and $\psi(t)$ be $C^{1}([a, b])$ functions which satisfy

$$
\frac{d \varphi}{d t}>F(t, \varphi(t)), \quad \frac{d \psi}{d t}=F(t, \psi(t)), \quad a \leq t \leq b,
$$

and $\varphi(a)=\psi(a)$, where $F \in C([a, b], R)$. Then $\varphi(t)>\psi(t)$ for $a<t \leq b$. If

$$
\frac{d \varphi}{d t}<F(t, \varphi(t)) \quad \text { and } \quad \varphi(a)=\psi(a)
$$

then $\varphi(t)<\psi(t)$ for $a<t \leq b$.

Proof. Evidently $\frac{d \varphi}{d t}(a)>\frac{d \psi}{d t}(a)$. Therefore $\varphi(t)>\psi(t)$ for $t \in(a, a+\varepsilon)$ for some positive $\varepsilon$. The graph of $\varphi(t)$ cannot cross the graph of $\psi(t)$ downwards. Therefore $\varphi(t)>\psi(t)$ for $t \in(a, b]$. 


\subsection{Angular functions}

In this subsection we follow the comparison results of [9, Ch. 15] adapting them to our cases.

Consider two second order equations written in a form of systems of two first order equations:

$$
\left\{\frac{d x}{d t}=y, \quad \frac{d y}{d t}=-q(x)\right.
$$

and

$$
\left\{\frac{d x}{d t}=y, \quad \frac{d y}{d t}=-\tilde{q}(x),\right.
$$

where $\tilde{q}(x)$ possesses the property of positive homogeneity, that is, $\tilde{q}(c x)=$ $c \tilde{q}(x)$ for $c \geq 0$ (in fact $\tilde{q}(x)$ is a piece-wise linear function defined separately for $x \geq 0$ and $x<0)$. Suppose that

$$
x q(x)>x \tilde{q}(x), \quad x \neq 0 .
$$

Introduce the polar coordinates $(x, y)=\left(x, x^{\prime}\right)$ as

$$
x(t)=r(t) \sin \varphi(t), \quad x^{\prime}(t)=r(t) \cos \varphi(t)
$$

and let $(r(t), \varphi(t)),(\tilde{r}(t), \tilde{\varphi}(t))$ be coordinates for (3.3), (3.4) respectively.

One gets for $\varphi(t)$ and $\tilde{\varphi}(t)$ that

$$
\frac{d \varphi}{d t}=\frac{1}{r}\left[r \cos ^{2} \varphi+q(r \sin \varphi) \sin \varphi\right] .
$$

On the other hand,

$$
\begin{aligned}
\frac{d \tilde{\varphi}}{d t} & =\frac{1}{\tilde{r}}\left[\tilde{r} \cos ^{2} \tilde{\varphi}+\tilde{q}(\tilde{r} \sin \tilde{\varphi}) \sin \tilde{\varphi}\right] \\
& =\cos ^{2} \tilde{\varphi}+\tilde{q}(\sin \tilde{\varphi}) \sin \tilde{\varphi}:=F(\tilde{\varphi})
\end{aligned}
$$

It follows from (3.5) that $q(r \sin \varphi) \sin \varphi>\tilde{q}(r \sin \varphi) \sin \varphi$ if $\varphi \neq \bmod (\pi)$ and therefore

$$
\frac{d \varphi(t)}{d t}>F(\varphi(t))
$$

and, if $\varphi(a)=\tilde{\varphi}(a)$, then, by Theorem $2, \varphi(t)>\tilde{\varphi}(t)$ for any $t \in[a, b]$.

If inequality (3.5) is changed to the opposite then

$$
\frac{d \varphi(t)}{d t}<F(\varphi(t))
$$

and, if $\varphi(a)=\tilde{\varphi}(a)$, then, by Theorem $2, \varphi(t)<\tilde{\varphi}(t)$ for any $t \in[a, b]$.

\subsection{Comparison of angular functions}

Consider shortened equation

$$
x^{\prime \prime}=-\lambda f\left(x^{+}\right)+\mu f\left(x^{-}\right)
$$


and compare it to equations (3.1) and (3.2) having in mind the relations (1.2).

Notice that for $\lambda>0, \mu>0$

$$
x\left(\lambda k x^{+}-\mu k x^{-}\right)>x\left(\lambda f\left(x^{+}\right)-\mu f\left(x^{-}\right)\right)>x\left(\lambda l x^{+}-\mu l x^{-}\right), \quad x \neq 0 .
$$

The right-hand sides of equations (3.1) and (3.2) are positive homogeneous functions, therefore the arguments of preceding subsection are applicable.

If $\varphi_{k}(t), \varphi(t)$ and $\varphi_{l}(t)$ are the angular functions for equations (3.1), (3.8), (3.2) respectively, one has that

$$
\varphi_{k}(t)>\varphi(t)>\varphi_{l}(t), \quad t \in(0,1]
$$

if $\varphi_{k}(0)=\varphi(0)=\varphi_{l}(0)$. Thus we have arrived to the following result.

Lemma 1. Let $(\lambda, \mu)$ be in $D(k)_{i} \cap D(l)_{i}$ for some $i \in\{0,1, \ldots\}$. Then the angular functions for equations (3.1), (3.7), (3.2), which satisfy

$$
\varphi_{k}(0)=\varphi(0)=\varphi_{l}(0)=\varphi_{0}, \quad \varphi_{0}=0 \quad \text { or } \quad \varphi_{0}=\pi
$$

satisfy also the inequalities (3.9).

Remark 1. The above lemma means that for $(\lambda, \mu) \in D(k)_{i} \cap D(l)_{i}$ any solution of equation (3.7) with the initial conditions $x(0)=0, x^{\prime}(0)>0$ has exactly $i$ zeros in $(0,1)$ and $x(1) \neq 0$. The same is true for solutions of equation (3.7) with the initial conditions $x(0)=0, x^{\prime}(0)<0$.

\subsection{Result}

Consider equation

$$
x^{\prime \prime}=-\lambda f\left(x^{+}\right)+\mu f\left(x^{-}\right)+h\left(t, x, x^{\prime}\right)
$$

and the equivalent system

$$
\left\{\frac{d x}{d t}=y, \quad \frac{d y}{d t}=-q(x)+h(t, x, y),\right.
$$

where $q(x)=\lambda f\left(x^{+}\right)-\mu f\left(x^{-}\right)$. Suppose polar coordinates $(\rho(t), \theta(t))$ are introduced as $x(t)=\rho(t) \sin \theta(t), x^{\prime}(t)=\rho(t) \cos \theta(t)$. The expression for $\theta(t)$ is given as

$$
\frac{d \theta}{d t}=\left[\rho \cos ^{2} \theta+q(\rho \sin \theta) \sin \theta-h(t, \rho \sin \theta, \rho \cos \theta) \sin \theta\right] / \rho .
$$

The right hand sides of equations (3.11) and (3.6) differ only by the term $\frac{1}{\rho} h(t, \rho \sin \theta, \rho \cos \theta) \sin \theta$, which is negligibly small if $\rho(t)$ stays in a complement of the circle of sufficiently large radius for any $t \in[0,1]$ (recall that $h$ is bounded). This is the case for the solutions of equation (3.10) which satisfy the initial conditions

$$
x(0)=0, \quad x^{\prime}(0)= \pm \Delta,
$$

if $\Delta \rightarrow+\infty$. For this, let us mention the following result. 
Lemma 2. For solutions of the problems (3.10), (3.12) a function $m(\Delta)$ exists such that $m(\Delta) \rightarrow+\infty$ as $\Delta \rightarrow+\infty$ and $\rho(t) \geq m(\Delta)$ for any $t \in[0,1]$.

Lemma follows from Lemma 15.1 in [9] since all solutions of equation (3.10) are extendable to the interval $[0,1]$. The latter follows from the assumptions on $f(1.2)$ and boundedness of $h$. It follows from the above arguments that

$$
\varphi_{k}(t) \geq \theta(t) \geq \varphi_{l}(t), \quad t \in[0,1]
$$

if $\varphi_{k}(0)=\varphi(0)=\varphi_{l}(0)$, where $\theta(t)$ is the angular function for solutions of (3.10), (3.12) with sufficiently large $\Delta$.

In other words, in conditions of Theorem 1 , a solution $\bar{x}(t)$ of equation (3.10) with the initial conditions $x(0)=0, x^{\prime}(0)=\Delta$ has exactly $i$ zeros in $(0,1)$ and $\bar{x}(1) \neq 0$. A solution $\underline{x}(t)$ of equation $(3.10)$ with the initial conditions

$$
x(0)=0, \quad x^{\prime}(0)=-\Delta
$$

also has exactly $i$ zeros in $(0,1)$ and $\underline{x}(1) \neq 0$. What is important, one has also $\bar{x}(1) \underline{x}(1)<0$. Then one concludes, considering the Cauchy problem (3.10),

$$
x(0)=0, \quad x^{\prime}(0)=\delta, \quad \delta \in(-\Delta, \Delta)
$$

and employing the continuous dependence of solutions on the initial data, that for some $\delta$ a solution $x(t)$ vanishes at $t=1$. This completes the proof of Theorem 1.

\section{Elementary Analysis of Regions $D_{i}$}

In order to analyze the regions $D(k)_{i}$ for equation $x^{\prime \prime}=-\lambda k x^{+}+\mu k x^{-}$recall that branches of the Fučík spectrum are given by

$$
\begin{aligned}
F_{0}^{+} & =\left\{(\lambda, \mu): \frac{\pi}{\sqrt{\lambda k}}=1, \mu \geq 0\right\}, \quad F_{0}^{-}=\left\{(\lambda, \mu): \lambda \geq 0, \frac{\pi}{\sqrt{\mu k}}=1\right\}, \\
F_{2 i-1}^{+} & =\left\{(\lambda ; \mu): i \frac{\pi}{\sqrt{\lambda k}}+i \frac{\pi}{\sqrt{\mu k}}=1\right\}, F_{2 i-1}^{-}=\left\{(\lambda ; \mu): i \frac{\pi}{\sqrt{\mu k}}+i \frac{\pi}{\sqrt{\lambda k}}=1\right\}, \\
F_{2 i}^{+} & =\left\{(\lambda ; \mu):(i+1) \frac{\pi}{\sqrt{\lambda k}}+i \frac{\pi}{\sqrt{\mu k}}=1\right\}, \\
F_{2 i}^{-} & =\left\{(\lambda ; \mu):(i+1) \frac{\pi}{\sqrt{\mu k}}+i \frac{\pi}{\sqrt{\lambda k}}=1\right\} .
\end{aligned}
$$

Similar formulas are true for equation $x^{\prime \prime}=-\lambda l x^{+}+\mu l x^{-}$.

A set $D(k)_{0}$ is a square below $F_{0}^{-}$and to the left of $F_{0}^{+}$. A set $D(k)_{1}$ is a region bounded by $F_{0}^{-}, F_{0}^{+}$and $F_{1}^{ \pm}$. A set $D(k)_{2}$ is a region bounded by $F_{1}^{ \pm}$ and $\min \left\{F_{2}^{+}, F_{2}^{-}\right\}$. A union of these regions is depicted in Fig. 3.

Similarly, regions $D(l)_{i}$ can be described. Since $l<k$, the spectrum $\Sigma_{F}(l)$ can be obtained from $\Sigma_{F}(k)$ by extension. Under the extension process

$$
D(k)_{0} \cap D(l)_{0}=D(k)_{0} \neq \varnothing .
$$




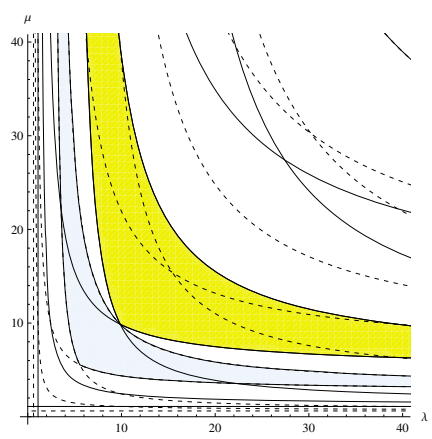

a)

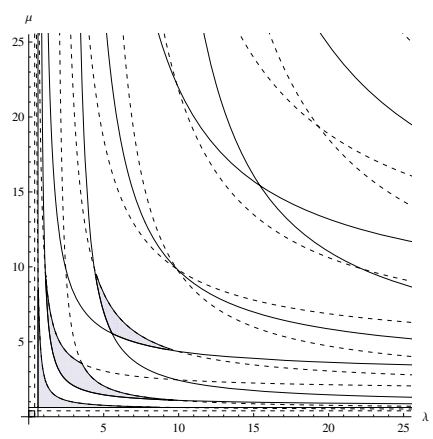

b)

Figure 3. Several first branches of the Fučík spectra for problems (3.1) (dashed curves) and (3.2) (solid curves) are given; a) $D(k)_{3}$ (on the left) and $D(l)_{3}$ for $k / l=(4 / 3)^{2}$ are shaded, and their intersection is empty (a "common" point does not belong to $D(k)_{3} \bigcap D(l)_{3}$ since both sets are open; b) "Good" regions $D(l)_{0} \bigcap D(k)_{0}, D(l)_{1} \bigcap D(k)_{1}$, $D(l)_{2} \bigcap D(k)_{2}$ and $D(l)_{3} \bigcap D(k)_{3}$ for $k / l=(5 / 4)^{2}$.

Therefore for any ratio $k / l$ the problem (1.1) is solvable if

$$
(\lambda, \mu) \in D(k)_{0} \cap D(l)_{0}=D(k)_{0} .
$$

Not the case for $i>0$. Generally, if $\frac{k}{l}$ is too large, the intersection of $D(k)_{i}$ and $D(l)_{i}$ is empty. The precise values of $k / l$ for any $i=1,2, \ldots$ are given below.

Proposition 1. If $1<k / l<(i+1 / i)^{2}$ then $D(k)_{i} \cap D(l)_{i} \neq \varnothing, i=1,2, \ldots$. If $k / l \geq(i+1 / i)^{2}$, then $D(k)_{i} \cap D(l)_{i}=\varnothing$.

The proof by elementary geometrical considerations.

Corollary 1. If $k / l \geq(i+1 / i)^{2}$ then $D(k)_{j} \cap D(l)_{j}=\varnothing$ for any $j \geq i$.

Therefore there exist only finite non-empty intersections $D(k)_{j} \cap D(l)_{j}$, if $k>$ $l>0$.

Corollary 2. If $k / l<(i+1 / i)^{2}$ then $D(k)_{j} \cap D(l)_{j} \neq \varnothing$ for any $j<i$.

\section{Example}

Consider the problem (1.1), where

$$
f(x)=\frac{3}{20} x\left(8+\frac{3 x \sin (5 x)}{1+x^{2}}\right), \quad h\left(t, x, x^{\prime}\right)=\frac{1}{1+t^{2} x^{\prime 2}} .
$$

Then conditions (1.2) are fulfilled with $l=0.8$ and $k=1.5$, see Fig. 4a).

Let $D(k)_{i}$ be a "good" region where the IVPs

$$
x^{\prime \prime}+\lambda k x^{+}-\mu k x^{-}=0, \quad x(0)=0, \quad x^{\prime}(0)= \pm 1
$$




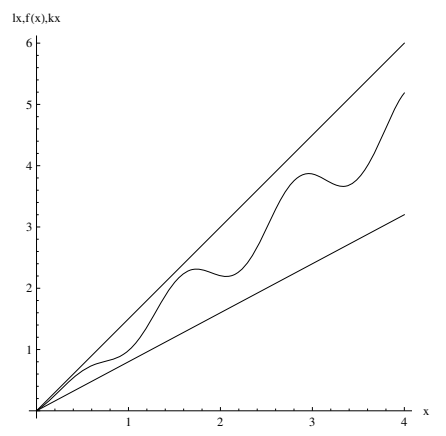

a)

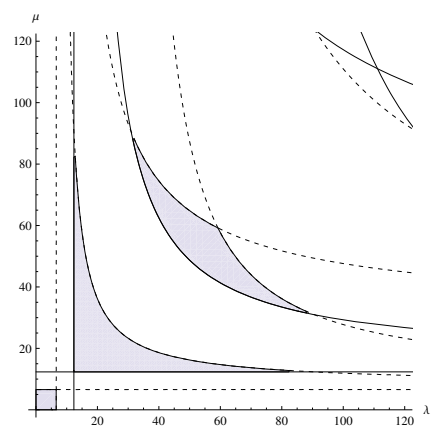

b)

Figure 4. a) The graphs of $f(x)$ and the linear functions $k x$ and $l x, k=1.5, l=0.8$; b) Intersections $D(l)_{i} \bigcap D(k)_{i}, k=0,1,2$.

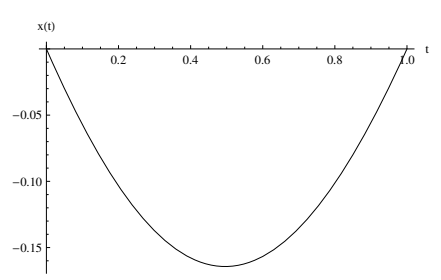

a)

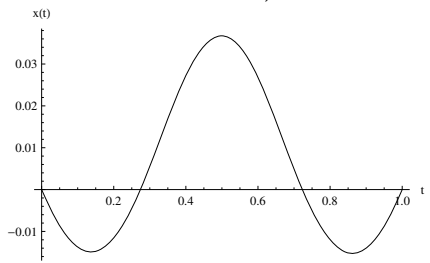

c)

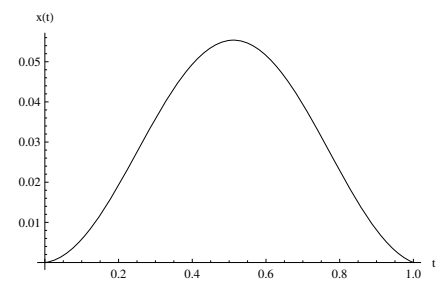

b)

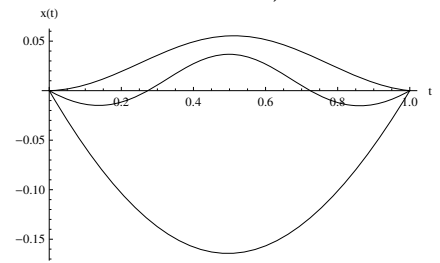

d)

Figure 5. Solutions of BVP (1.1) where $f$ and $h$ are as in Example: a) $\left.\left.(\lambda, \mu)=(4,2) \in D_{0}, x^{\prime}(0)=-0.627431 ; \mathrm{b}\right)(\lambda, \mu)=(30,15) \in D_{1}, x^{\prime}(0)=0.0103358 ; \mathrm{c}\right)$ $(\lambda, \mu)=(70,40) \in D_{2}, x^{\prime}(0)=-0.201086$. d) Three solutions of BVP (1.1).

have solutions with exactly $i$ zeros in $(0,1)$ and these solutions have opposite signs at $t=1$.

There are countably many "good" regions $D(k)_{i}$ and $D(l)_{i}$ but only three intersections $D(k)_{i} \cap D(l)_{i}$ are non-empty, namely, for $i=0,1,2$, see Fig. $\left.4 \mathrm{~b}\right)$. The corresponding solutions of BVP (1.1) are depicted in Fig. 5.

\section{Conclusions}

The problem

$$
x^{\prime \prime}=-\lambda x^{+}+\mu x^{-}+h\left(t, x, x^{\prime}\right), \quad x(0)=0, \quad x(1)=0
$$


is solvable if $(\lambda, \mu)$ is in one of "good" regions (with respect to the Fučík spectrum $\Sigma_{F}$ ) depicted in Fig. 2 and $h$ is bounded. There are infinite number of "good" regions.

The same is true for the problem

$$
x^{\prime \prime}=-\lambda f\left(x^{+}\right)+\mu f\left(x^{-}\right)+h\left(t, x, x^{\prime}\right), \quad x(0)=0, \quad x(1)=0,
$$

where $l x<f(x)<k x$ and some technical assumptions (mentioned in Theorem 1) are in force. The essential difference is that the number of "good" regions is always finite. If $k$ is significantly greater than $l$ then only one "good" region $D_{0}$ exists.

\section{References}

[1] C. De Coster and P. Habets. A two parameters Ambrosetti-Prodi problem. Port. Math., 53(3):279-303, 1996.

[2] W. Dambrosio. Qualitative results for parameter dependent asymmetric oscillators. Nonlinear Anal., 47(7):5021-5028, 2001. http://dx.doi.org/10.1016/S0362-546X(01)00613-7.

[3] S. Fučík and A. Kufner. Nonlinear Differential Equations. Elsevier, 1980.

[4] A. Gritsans and F. Sadyrbaev. Nonlinear spectra for parameter dependent ordinary differential equations. Nonlinear Anal. Model. Control, 12(2):253-267, 2007.

[5] A. Gritsans and F. Sadyrbaev. On nonlinear Fučík type spectra. Math. Model. Anal., 13(2):203-210, 2008. http://dx.doi.org/10.3846/1392-6292.2008.13.203-210.

[6] A. Gritsans and F. Sadyrbaev. Two-parametric nonlinear eigenvalue problems. In L. Hatvani and T. Krisztin(Eds.), Proc. of the 8th Colloquium on the Qualitative Theory of Differential Equations, Szeged, Hungary, 2007, pp. 1-14, 2008.

[7] A. Gritsans and F. Sadyrbaev. Nonlinear spectra: the Neumann problem. Math. Model. Anal., 14(1):33-42, 2009. http://dx.doi.org/10.3846/1392-6292.2009.14.33-42.

[8] A. Gritsans and F. Sadyrbaev. Two-parameter nonlinear oscillations: the Neumann problem. Math. Model. Anal., 16(1):23-38, 2011. http://dx.doi.org/10.3846/13926292.2011.559449.

[9] M. Krasnoselskii, A. Perov, A. Povolockii and P. Zabreiko. Plane Vector Fields. Academic Press, 1966. (in Russian)

[10] A.C. Lazer and P.J. McKenna. Large-amplitude periodic oscillations in suspension bridges: some new connections with nonlinear analysis. SIAM Rev., 32(4):537-578, 1990. http://dx.doi.org/10.1137/1032120.

[11] F. Sadyrbaev. Multiplicity in parameter-dependent problems for ordinary differential equations. Math. Model. Anal., 14(4):503-514, 2009. http://dx.doi.org/10.3846/1392-6292.2009.14.503-514.

[12] T. Shibata. Two-parameter nonlinear Sturm-Liouville problems. Proc. Edinb. Math. Soc., 41(2):225-245, 1998. http://dx.doi.org/10.1017/S0013091500019611. 